\title{
Research on Open Virtual Simulation Experiment Teaching Model Based on Cloud Platform in Network Security
}

\author{
Liu Li ${ }^{1, a}$, Guoqiang $\mathrm{Li}^{1, b}$, Linhua Ding ${ }^{1, \mathrm{c}}$ and Jiuru Wang ${ }^{1, \mathrm{~d}}$ \\ ${ }^{1}$ College of Information Science and Engineering, Linyi University, Shandong, China \\ axiaoke_1981@126.com, bliguoqiang@lyu.edu.cn, 'dinglinhua@lyu.eud.cn, dwangjiuru@lyu.eud.cn \\ * Liu Li
}

Keywords: Cloud Platform; Openness; Virtual Simulation; Network Security.

\begin{abstract}
Under the guidance of the orientation of innovative and entrepreneurial comprehensive universities with distinct regional characteristics, this paper fully investigates and analyses the employment direction data of students majoring in network engineering. According to the characteristics of students majoring in network engineering, this paper investigates the actual work needs of network security companies and enterprises and institutions for network security maintenance. The open virtual simulation experiment teaching mode of cloud platform is introduced into the teaching of network security course. It integrates the teaching content with the real environment of the enterprise, enables students to participate in the whole process of the experiment project, and takes the competition mode as the strong support of the project-oriented mode, aiming at improving students'interest in learning and the teaching effect of this course.
\end{abstract}

\section{基于云平台的开放性虚拟仿真实验教学模式在《网络安全》中的研究}

\author{
刘丽 $1, a$, 李国强 $1, b$, 丁林花 ${ }^{1, c}$, 王九如 ${ }^{1, d}$ \\ 1临沂大学信息科学与工程学院, 临沂, 山东, 中国
}

axiaoke_1981@126.com, bliguoqiang@lyu.edu.cn, 'dinglinhua@lyu.eud.cn, dwangjiuru@lyu.eud.cn *刘丽

关键词：云平台; 开放性；虚拟仿真；网络安全

中文摘要. 本文在区域特点鲜明的创新创业型综合性大学的定位指导下，充分调研和分析网 络工程专业的学生就业方向数据, 根据网络工程专业的学生特点, 实地考察当前网络安全公 司和企事业单位网络安全维护的实际工作需求, 将基于云平台的开放性虚拟仿真实验教学模 式引入到《网络安全》课程教学中, 将教学内容与企业真实环境融合, 让学生参与实验项目 全过程, 并将竞赛模式作为项目导向模式的有力支撑, 旨在提高学生学习兴趣, 提高该课程 教学成效。

1. 引言

《网络安全》课程是一门实践性很强的课程，如果空有理论，没有实践，对于将来走向 工作岗位是没有用处的, 又鉴于网络安全实验都具有一定的危险性, 网络安全教学受到了很 大的限制。当前大多数高校实验室的PC 上大都安装了硬盘还原保护卡，在一定程度了加强 了对电脑的保护，但是也给网络安全实验带来了很多难题，学生无法使用那些安装后需要操 作系统重新引导的实验软件, 可是如果不安装还原卡, 病毒木马实验如果控制不好, 就会造 
成严峻的后果, 最后导致学生对实验过程只能通过简单的文字描述了解,对其工作原理不能获 得深刻的理解。后来各个高校开始探索虚拟仿真实验平台, 其中有些大学已经成功开发了《数 字图像处理》、《计算机组成原理》等多个虚拟实验系统，一些大学现代教育技术研究所研制 的基于虚拟空间环境的三维电子线路实验系统 EVLab 是一个基于虚拟空间的三维电子线路 实验环境, 但是这样的实验会造成比较古老, 内容落后。

本文将基于云平台的开放性虚拟仿真教学模式引入到《网络安全》课程的实验教学中, 以网络安全公司和企事业单位的网络安全维护中真实项目为依托, 培养学生的动手实践能力、 应用能力和创新能力; 同时这些实验与实际联系密切, 从而提高学生的学习兴趣。

\section{2. 传统教学模式}

各个高校的网络安全实验课程都在积极探索新的教学模式，尤其是基于虚拟仿真环境在 线实验教学模式得到了各大高校的重视和实际应用。但是目前已有的教学模式中存在以下问 题:

(1) 目前有关网络安全实验教学改革方面的研究成果基本上都是进行教学内容和教学方 法等方面的改革，但是这些改革探索没有注重该课程内容与现实网络安全发展之间的区别与 联系, 跟不上知识更新换代的步伐, 从而使得这些改革举措只是在某个教学内容模块中有效。

（2）由于很多网络安全很多实验不能在真机中演练，很多攻击防御技术只停留在理论空 想阶段, 目前很多高校也都采用了虚拟仿真平台来做网络安全实验, 虚拟仿真平台可以不需 要网络环境, 单机就可以运行, 做一些有关木马病毒的实验比较安全, 但是实验更新不及时, 不能让学生掌握最新的技术，导致走上工作岗位后不能及时适应岗位所需。

（3）基于云平台的虚拟仿真实验教学模式可以在实验课程中取得良好的教学效果，但是 目前该模式由于实验内容教难, 理论教深, 如果只联系实际, 而不注重即使更新理论的传授, 使得学生也很难接受。再加上针对本课程的开放实验云平台基本上都是收费的, 所以很多高 校仍然不想去使用。

（4）大部分已经存在的基于云平台的虚拟仿真实验都不能满足在线和大规模容量的需 求, 也受到了场地、环境的影响, 还不能实时的教学互动。

\section{3. 改进的教学模式}

《网络安全》课程的实验内容复杂多变, 我们不可能在本机直接实验种植木马, 植入病 毒, 也不可能去实际的进行暴力破解及入侵。导致很多知识的讲解仅仅停留在理论空想阶段, 即便是有实验可做, 也比较古老, 在某种程度上降低了学生的学习兴趣, 也引入过虚拟仿真 平台, 里面的实验内容更新不及时, 所以只能寻求别的实验方法。

在本教学模式中, 我们引入了在线实验云平台开放性实验模式, 把《网络安全》的实验 课程教学内容分成三个级别: 初级 (与课本内容紧密相关) 、中级 (需要扩展知识) 和高级 模块（需要一定的实战能力）, 对于基础弱的学生来说, 可以先从初级实验为开始, 当有了 一定的实战经验和理论知识, 就可以去做中级实验, 高级实验属于选做实验, 感兴趣的同学 可以去演练, 这些同学可以通过做这些高级实验提高自己的实战能力, 为参加全国网络安全 大赛最准备。整个的实验采用MOOE模式, 环境之间采用物理隔离随时中断学习自动保存学 习进度, 不局限于地点和时间, 在宿舍或者其他地方都可以完成。

具体的改进措施表现在以下几个方面:

\section{1 采用MOOE模式}

MOOE（Massive Open Online Experiments）是一种 “大规模在线开放实验” 模式，它采 用虚拟化与 $\mathrm{SDN}$ 等技术，使用先进的软件定义网络与共享硬件资源思想，解决了传统实验室 在时间、空间与实验内容等限制, 例如高校一个班级学生大约 70 人, 如果好几个班级同时实 
验的话, 服务器若不够稳定, 在实验过程中很容易导致服务器瘫疾, 影响课程的进行, 如果 云平台采用了MOOE模式，可以使得同时大量学生在线而能提供正常的服务。

采用MOOE模式, 是本课程虚拟实验平台的最大优势, 这个模式有两大优点: 一是环境 之间采用物理隔离, 学生实验可以在上课时完成, 对于复杂实验或者高级实验课堂上没法完 成的, 可以随时中断学习自动保存学习进度, 在宿舍或者其他有电脑的地方, 让学生在虚拟 的真实开发环境下, 通过实验操作方式学习网络安全技术。二是具有 “大规模” 的特点, 对 于好多学生同时上课也不会有因为端口而冲突, 更不会因为人数增多而使得系统崩溃。如图1 运行界面所示:

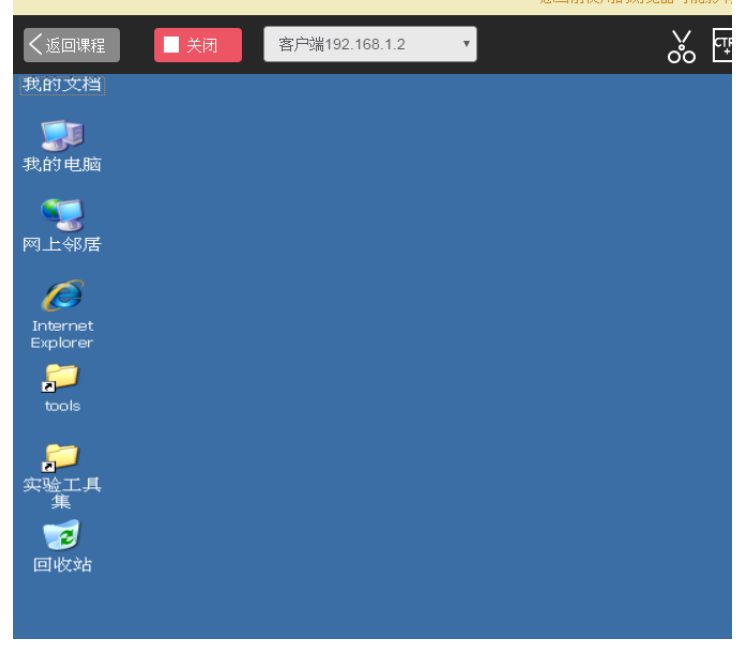

图1 运行界面

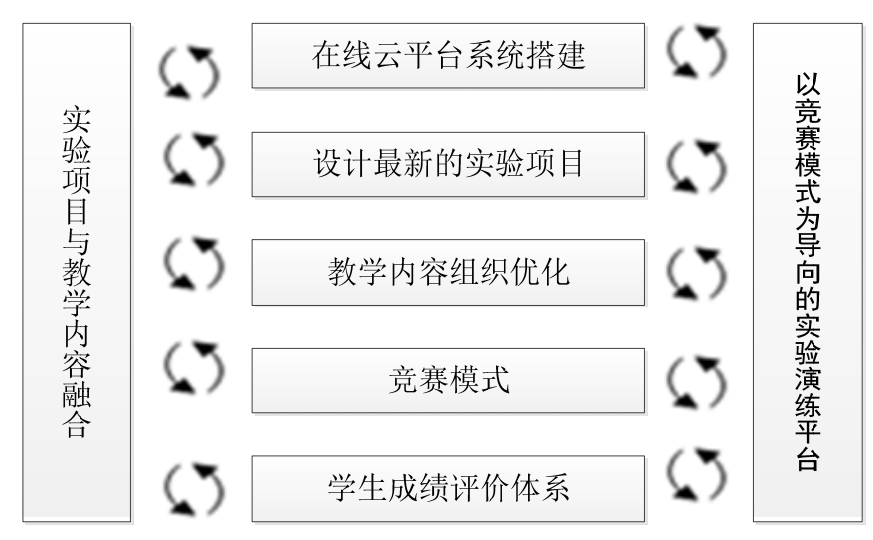

图2 融合内容

\section{2 采用虚拟仿真模式}

虚拟仿真是网络安全课程经常用到的实验教学模式, Vmware虚拟机就是虚拟仿真实验的 一个典型代表, 它是一个虚拟出来的环境, 在这个环境里实验就跟在真机中实验室类似的, 但是单纯的虚拟机只能模拟一台单机, 像防火墙、路由器等这样的大型设备必须嵌入才可以, 后来就在虚拟机的基础上开发了虚拟仿真环境。使用户可直接参与并探索仿真对象在所处环 境中的作用与变化。

如果实验课堂采用了虚拟仿真模式, 在此模式中搭建了各种各样的服务, 学生就像在真 实的环境中做实验一样, 例如木马、病毒实验都可以在这个环境中去做。而不必担心会传染, 会破坏系统。在实验中, 可以采用小组团队协作的方式, 一方面提高了学生的自主学习能力 和创新能力, 另一方面也提升了学生的团队合作能力和协调能力。

\section{3 实验教学内容加课堂内容的融合}

网络安全很多技术如加密技术，木马技术，黑客攻击技术，入侵检测技术等都很难懂， 涉及的知识面广泛, 具有跨学科的特点, 涉及到了计算机网络, 程序算法还有一些离散数学 的知识甚至一些心理学等课程, 而课时有限, 不可能面面俱到, 讲解时要有重点, 围绕关键 技术 “精讲多练”。另外, 仅利用有限的理论教学学时和实验教学学时学生难以掌握该课程 的重点, 课外自主学习也尤为关键。因此, 教学内容组织优化主要就是指实验教学实验项目 的选取设计和课外学习平台与学习资源的融合。

在本实验平台上, 通过合理编排实验教学项目内容和教学过程, 精心设计在线实验项目, 使整个教学过程即能够激发学生的学习兴趣, 又能培养学生的职业素养和创新能力。融合内 容如图2所示: 


\section{3 综合测试与竞赛模式结合}

国内对各大高校开放的实验云平台有很多，中英文的都有，可以从中进行选择或自主搭 建。但是云平台的选择不应随意, 应该考虑到本院学生的实际情况和接受能力; 同时实验题 目的设计和选择是一个重点研究内容，既要基于学生当前的实际水平，还要考虑到技术的实 际应用型和趣味性, 还要考虑是否能培养学生的创新能力和团队协作能力。为了培养学生兴 趣, 实验课堂引入竞赛模式, 学生在学习知识的同时, 还能发起挑战, 启发学习兴趣。为了 培养学生创新能力, 实验课程教学项目需与网络安全公司真实技能相融, 学生通过分组参与 整个教学设计和教学过程, 体现 “学中做, 做中学” 的创新理念。

\section{4. 结束语}

国内对各大高校开放的实验云平台有很多，中英文的都有，可以从中进行选择或自主搭 建。但是云平台的选择不应随意, 应该考虑到本院学生的实际情况和接受能力; 同时实验题 目的设计和选择是一个重点研究内容, 既要基于学生当前的实际水平, 还要考虑到技术的实 际应用型和趣味性, 还要考虑是否能培养学生的创新能力和团队协作能力。为了培养学生兴 趣, 实验课堂引入竞赛模式, 学生在学习知识的同时, 还能发起挑战, 启发学习兴趣。选择 一个完全贴近实战的演练平台，在寓教于乐中磨练技艺，提高实战技能。

为了培养学生创新能力, 实验课程教学项目需与网络安全公司真实技能相融, 学生通过 分组参与整个教学设计和教学过程, 体现 “学中做, 做中学” 的创新理念。

\section{致谢}

本文为临沂大学教学质量工程项目 《基于云平台的开放性虚拟仿真实验教学模式在 “网 络安全” 中的研究研究》的阶段性成果之一; 山东省高等教育本科教改项目 《地方高校基于 校企协同的新工科专业改造升级探索与实践-以软件工程为例》(M2018X058)的阶段性成果之 一; 临沂大学在线课程《黑客攻与防》的阶段性成果之一。

\section{References}

[1] Hailong Wang. Exploration of Case Teaching Method in Network Security Experiment Teaching [J]. Suzhou Gaobo Vocational College of Software Technology, 2012, 7 (3)

[2] Fuhang Qiu, in-depth and simple explanation of the course "Network Security" [J]. Journal of Science and Technology, Changchun University, 2015 (9): 57-59.

[3] Lili Yan, Changyan, Zhang Shibin. Teaching Reform and Practice Exploration of Network Security Technology Course [J]. Computer Education, 2016 (2): 114-115.

[4] Yingjie Li. Effective Application of Virtual Simulation Experiment in Computer Network Teaching [J]. Information and Computer, 2011-1:169-176.

[5] Jianzhong Zhu. A Preliminary Study on Large-scale Online Open Experiment: Taking Network Security as an Example [J]. Journal of Henan Radio and Television University, 2016(1): 157-162. 